\title{
MARANHITES, UM IMPORTANTE FÓSSIL INDICE DO DEVONIANO SUPERIOR (ACRITARCHA SCUTELLOMORPHITAE)
}

\author{
por \\ IGNÄCIO MACHADO BRITO \\ Instituto de Geociências da Universidade Federal do Rio de Janeiro \\ Pesquisador I "A" do Conselho Nacional de Desenvolvimento Científico e Tecnológico
}

\section{RESUMO}

O gênero Maranhites é um importante fóssil do Devoniano. As formas dotadas de grande número de estruturas periféricas são exclusivas do Devoniano Superior, atingindo a base do Carbonífero. Aquelas com menos de quinze elementos periféricos, embora abundantes no Devoniano Superior, atingem o Devoniano inferior. As espécies mais abundantes, comuns ao Devoniano Superior de todas as bacias paleozóicas do Brasil, já foram assinaladas, dentre outras localidades, no sul de Gana e no Saara da Argélia.

\section{SUMMARY}

The genus Maranhites is an important Devonian index fossil. The forms with numerous peripherical structures are exclusive of the Upper Devonian up to the Lower Carboniferous. Those forms with less than fifteen peripherical elements, though frequent in the Upper Devonian, are found beginning at the Lower Devonian. The most abundant species, common in the Upper Devonian of all the Brazilian paleozoic basins were already registered, besides other localities, in Southern Ghana and the Algerian Sahara.

\section{KURZFASSUNG}

Die Gattung Maranhites ist ein wichtiges devonisches Leitfossil. Die Formen mit vielen Randstrukturen kommen nur im Oberdevon bis an die Basis des Karbons vor. Die Figuren mit weniger als fünfzehn Randelementen, obwohl sie im Oberdevon häufig sind, finden sich ab Unterdevon. Die häufigsten Arten des Oberdevons der brasilianishen palaeozoishenBecken wurden schon im Süden Ghanas und in der Sahara von Algier gefunden.

O gênero Maranhites foi proposto por BRITO (1965-A, p. 1) para microfósseis marinhos do Devoniano Superior das Bacias do Maranhão e do Jatobá. A descrição original, publicada num periódico de pequena circulação, foi a seguinte: "Grãos de contorno arredondado medindo de 80 a 150 micra, com um bordo geralmente ondulado, constituído de pequenos escudos entre os quais aparentemente podem ser observadas abertu- 


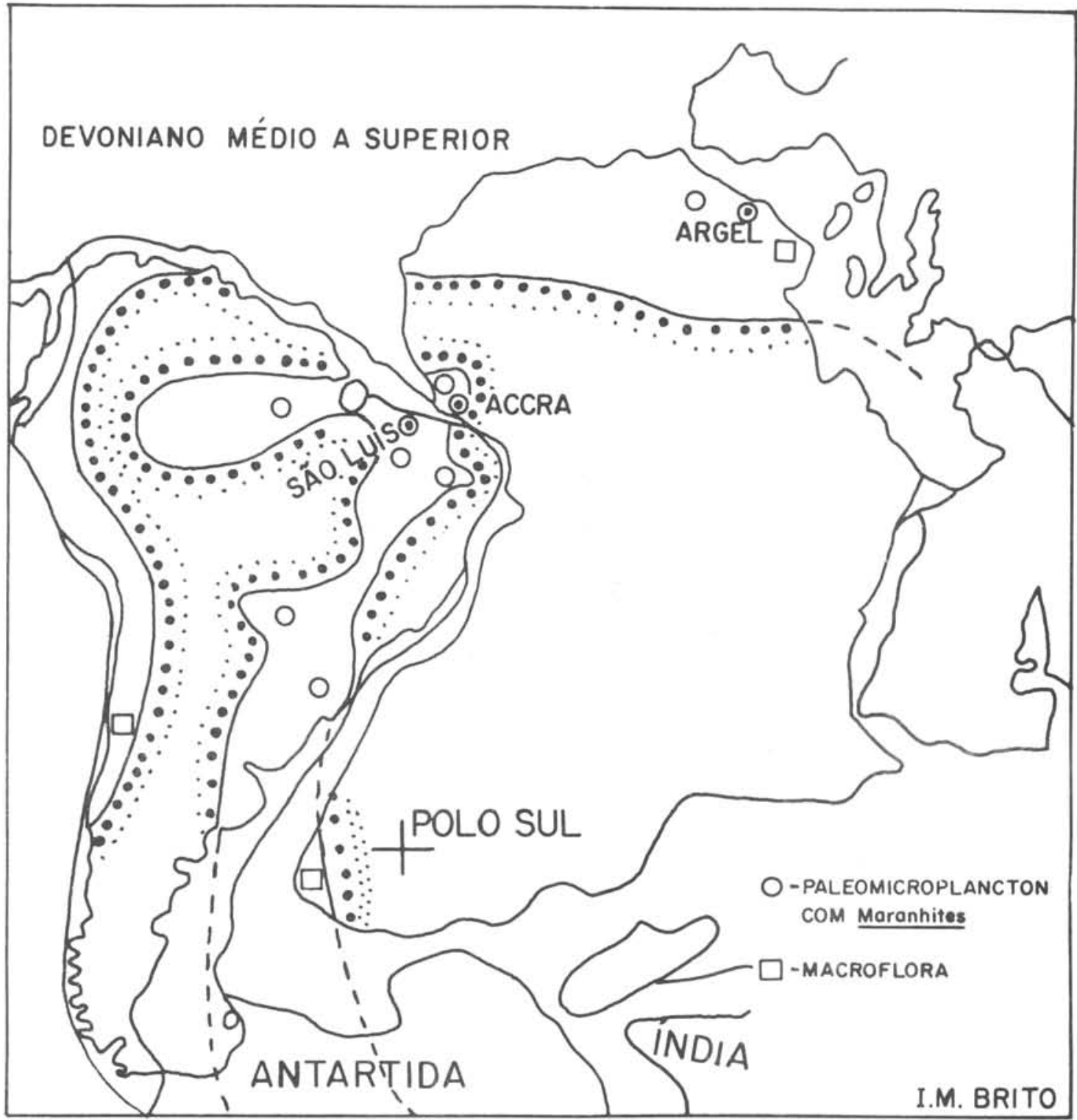

FIG. 1

Mapa Paleográfico

ras de pequeno tamanho. A principal característica é um escudo marginal bem maior que as demais figuras de ornamentação. Raramente são observados dois desses escudos diametralmente opostos. O referido escudo marginal aparentemente mostra-se como se fosse formado por três a cinco dos escudos marginais fundidos numa só peça. Os grãos apresentam dois planos de simetria, um passando pelo centro do escudo lateral grande e pelo centro do corpo do organismo e outro pelo plano equatorial. Os grãos, quando vivos deveriam ser oblatos e não esféricos. É comum a presença de uma auréola central, com estrias radiadas de coloração um pouco mais escura que o restante do grão que, com luz transmitida, apresenta-se amarelo castanho claro. Algumas formas apresentam características intermediárias entre Maranhites típico e Tapajonites mosesi Sommer" 
Tipo do gênero: Maranhites brasiliensis apresentado no trabalho como espécie nova, cuja descrição original é a seguinte (BRITO, 1965-A, p. 2):

Grãos arredondados com cerca de 150 micra de diâmetro. Os pequenos escudos situados no bordo mostram-se arredondados, ligados entre si por pequenos arcos e apresentam o diâmetro cerca de 15 vezes maior que o do grão. O escudo lateral é cerca de 4 vezes maior que os pequenos escudos marginais. Na parte central do corpo do grão uma auréola de coloração mais escura coberta em sua parte central por um retículo".

Localidade e estrato típico: Sondagem Slst-1-Ma, Test. 51, Zona palinológica Q superior.

O microfóssil foi considerado como Algae Incertae Sedis.

No mesmo ano, o autor (BRITO, 1965 -B, p. 4) afirma que representantes de $M$. brasiliensis são abundantes nos folhelhos de Ibimirim (bacia do Jatobá) e apresenta algumas fotografias dos mesmos.

Ainda o mesmo pesquisador (BRITO, 1967-B), num estudo detalhado sobre o gênero em questão, verificou que a testa desse microfóssil era oca e que os escudos perifericos eram estruturas internas. Classificou no gênero em questão a espécie Tasmanites mosesi Sommer, depois incluída por Sommer e Van Boekel no gênero Tapajonites, e descreveu uma nova espécie, também do Devoniano Superior da Bacia do Maranhão, que denominou de Maranhites pulcher.

DAEMON, QUADROS e SILVA (1967) apresentam, num estudo palinológico do Devoniano da Bacia do Paraná, as três espécies como formas gradacionais de Maranhites brasiliensis.

LUDWIG e MÜLLER (1968) também assinalam Maranhites brasiliensis em camadas do Devoniano Superior, atingindo a base do Carbonifero, na Bacia do Maranhão.
DAEMON (1974), classificando palinomorfos guias do Devoniano Superior e Carbonifero Inferior das Bacias do Amazonas e do Maranhão, apresenta a ocorrência de Maranhites na formação Curuá, de idade frasniana a tournaisiana e confirma a presença do mesmo na formação Longá e parte basal da Poti.

No exterior, o gênero foi assinalado por MOREAU-BENOIT (1966) no Devoniano Inferior de Anjou, através da espécie Maranhites mosesi (Sommer), descrita originalmente da formação Curuá da Bacia Amazônica.

STOCKMANS e Williêre (1969) descrevem uma nova espécie de Maranhites do Fameniano Inferior (Devoniano Superior) da Bélgica, que denominaram $M$. britoii JARDINÊ (1972) assinala no Saara da Argélia, em camadas do Devoniano Superior (Frasniano e Fameniano), as duas espécis brasileiras mais comuns e BÄR e RIEGEL (1974) noticiam a presença de Maranhites brasiliensis no Frasniano de Gana.

\section{SISTEMÂTICA GRUPO ACRITARCHA Evitt SUBGRUPO SCUTELLOMORPHITAE Brito, 1967-B}

Microfósseis com a testa arredondada, oca, oblata, geralmente lisa, com a periferia muitas vezes ondulada causada por estruturas internas em forma de colunas baixas e largas, próximas da margem. Essas estruturas podem estar unidas entre si por pequenas paredes e muitas apresentam uma de suas unidades de três a cinco vezes mais larga que as demais. Dois planos de simetria são observados, um no plano equatorial e outro passando pelo centro do corpo e pela unidade modificada quando esta é observada.

O subgrupo é representado, até o presente pelo gênero Maranhites Brito.

ESTAMPA I

Fig. 1-6 - Maranhites mosesii (Sommer) mostrando a variação do número de estruturas periféricas, de 3 (fig. 1) a 11 (fig. 3). Nas figuras 4 e 5 observa-se, com a entrada de água dentro do fóssil, que as estruturas são internas e não escudos externos. Com exceção da fig. 5 ( $\times 500)$, todas são aumentadas 300 vezes.

Fig. 7 - Maranhites pulcher Brito ( $\times 300)$.

Fig. 8-9 - Formas dẻ ?Maranhites com as estruturas periféricas não individualizadas ( $\times 250$ ).

Figs. 10-14 - Maranhites brasiliensis Brito mostrando a variação nas suas formas. Figs. 10-12 (x 250); Figs. 13-14 ( $\times 500)$. 

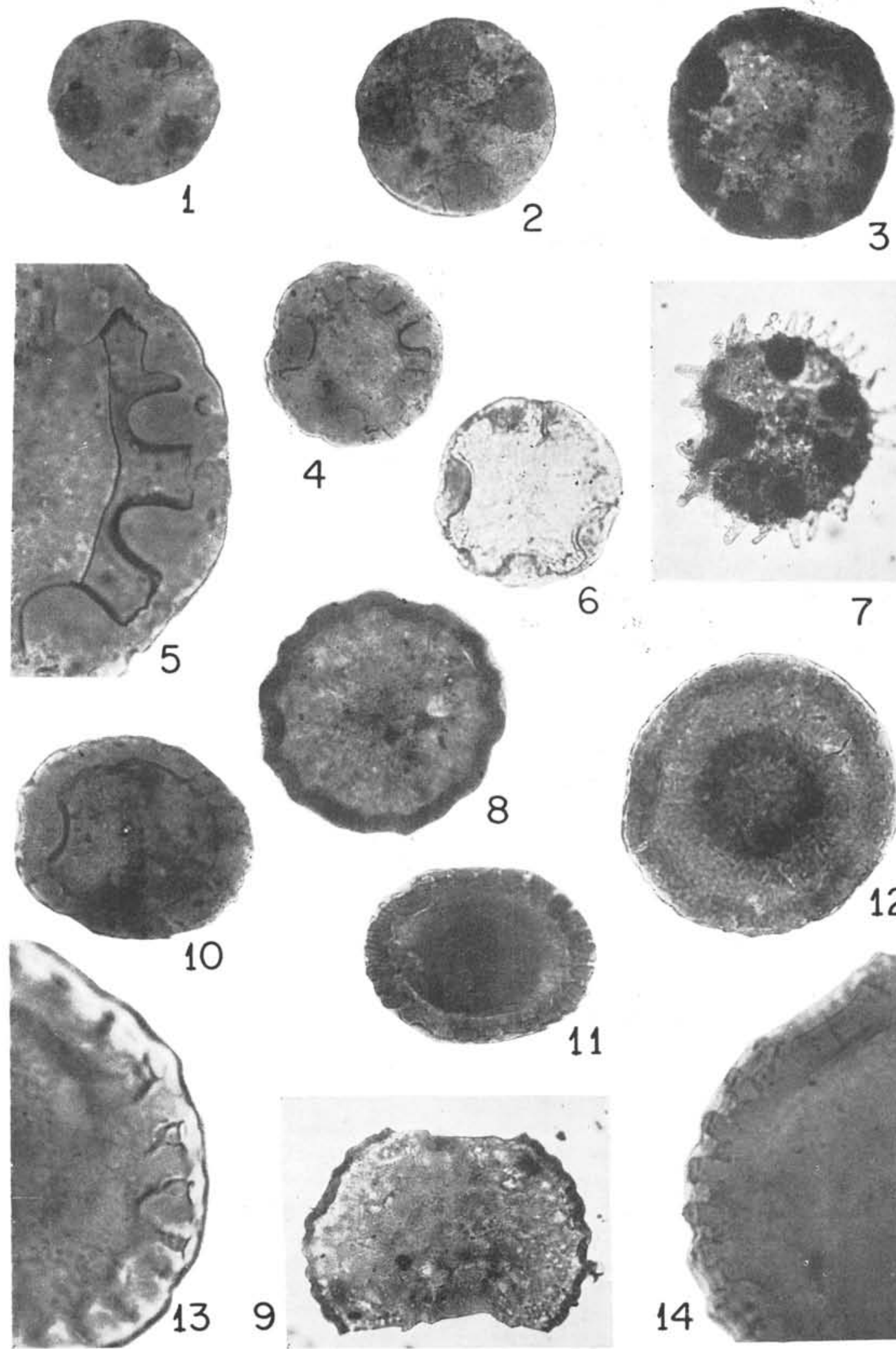

10
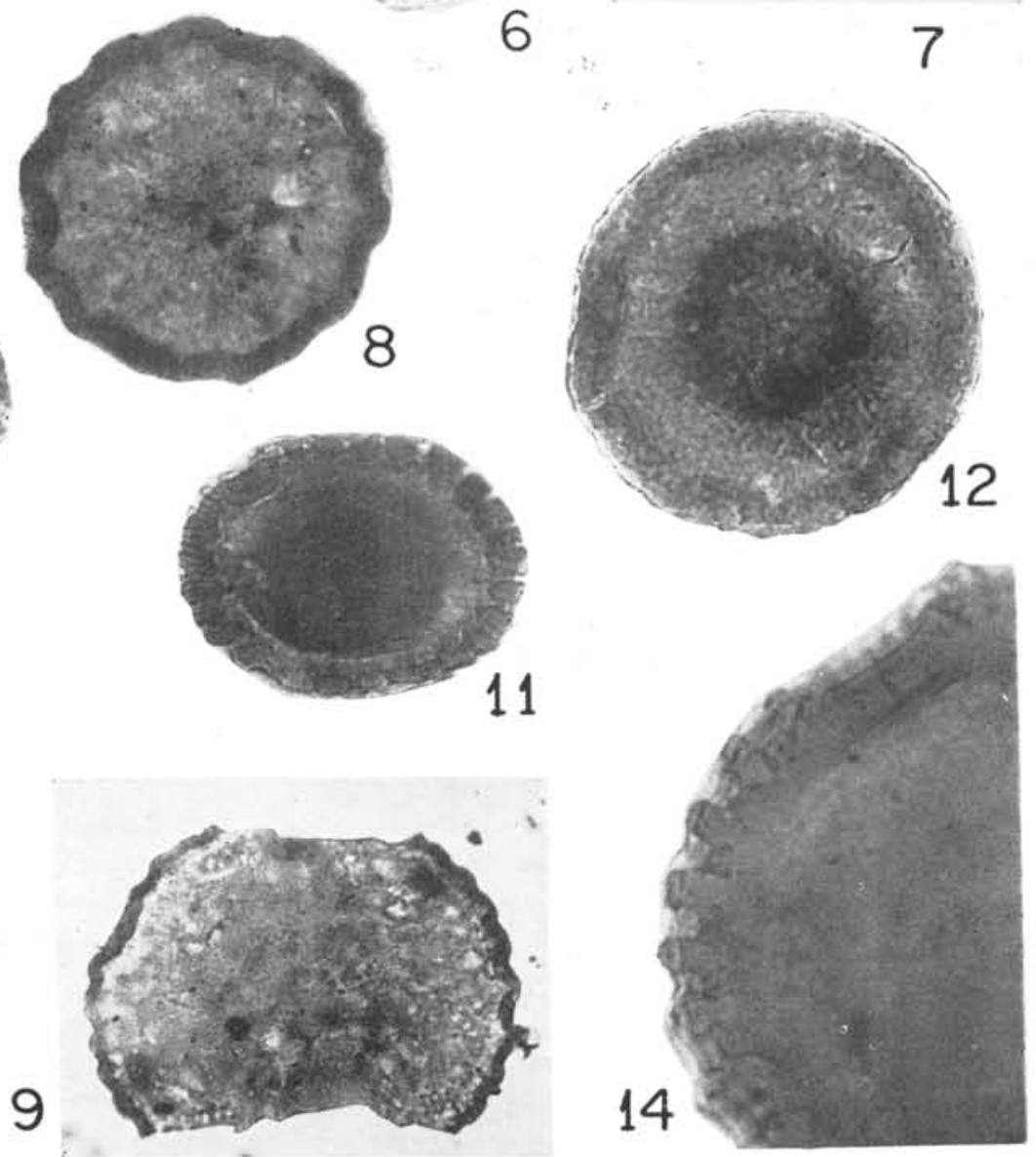


\section{GÊNERO Maranhites Brito, 1965-A}

Já apresentamos no início do presente trabalho a descrição original do gênero, assim como as ocorrências do mesmo. Enquanto o subgrupo Scutellomorphitae for representado exclusivamente pelo gênero em questão sua descrição pode ser exatamente a mesma de Maranhites que é representado, de acordo com os conhecimentos atuais, por quatro espécies.

\section{Maranhites brasilienses Brito, 1965-A}

Est. I, Figs. 10-14

Para sinomínia, vide BRITO, 1976, p. 753.

Esta espécie, cujo diâmetro atinge os 150 micra, é caracterizada pelo grande número de estruturas periféricas internas, geralmente acima de vinte, e por uma estrutura lateral cerca de quatro vezes mais larga que as demais.

A espécie já foi encontrada do Frasniano à base do Carbonifero Inferior das Bacias do Maranhão e do Amazonas, Frasniano das Bacias do Jatobá, Paraná e Sul de Gana e Frasniano e Fameniano do Saara da Argélia.

\section{Maranhites britoii Stockmans e Willière, 1969}

Maranhites britoii Stockmans e Willière, 1969, Mem. Acad. Royale Belgique, V. 38, fasc. 6 , p. 44 , Pl. II, figs. 4,7 .

Esta espécie, cujo diâmetro atinge 75 micra, apresenta cerca de quinze estruturas periféricas arredondadas, mas com tendências às formas quadradas ou retangulares. A estrutura lateral lembra a da espécie anterior.

$\mathrm{Na}$ descrição original, os seus autores comentaram que os exemplares estudados geralmente estavam incompletos. Muito curioso é a aparência escura desses microfósseis em contraste com as estruturas periféricas claras, exatamente o contrário das formas brasileiras.
A espécie foi descrita do Fameniano inferior da Bélgica.

\section{Maranhites mosesii (Sommer, 1956)}

Est. I, figs. 1-6

Para sinominia vide BRITO, 1976, p. 753

Microfósseis arredondados medindo cerca de 50 a 100 micra de diâmetro com as estruturas periféricas em número geralmente inferior a dez, isoladas umas das outras. Não apresentam estrutura lateral modificada. Tem praticamente a mesma distribuição de $M$. brasiliensis, mas ainda não foi encontrado no Saara e já foi assinalado no Devoniano do Peru e no Devoniano Inferior de Anjou, França.

Maranhites pulcher Brito, 1967

Est. I, fig. 7

Maranhites pulcher Brito, 1967, An. Acad. Brasil. Cienc., V, 39, n. 1, p. 165, Est. II, figs. 1, 2.

O corpo principal dessa espécie tem todas as características de $M$. mosesii (Sommer), mas apresenta um veu quase transparente no plano equatorial dotado de projeções digitiformes irregulares.

Já foi encontrado no Devoniano Superior da Bacia do Maranhão, de onde foi descrito originalmente, e no Devoniano Superior da Bacia do Paraná.

\section{CONCLUSÕES}

O Gênero Maranhites parece ser um excelente indicador do Devoniano marinho. As formas dotadas de um elevado número de estruturas laterais tais como $M$. Brasiliensis e $M$. britoi são exclusivas do Devoniano Superior, atingindo a base do Carbonífero Inferior e apresentando o máximo do seu desenvolvimento no Frasniano. $M$. mosesii, abundante no Devoniano Superior, é conhecido também em camadas atribuídas ao Devoniano Inferior. 


\section{BIBLIOGRAFIA}

BAR, P. \& RIEGEL, W.,-1974-Les Microflores des Séries Paléozoiques du Ghana (Afrique Ocidentale) et leurs relations paléofloristiques. Sci. Geol., Bull. 27, 1-2, p. 39-58, 2 est., 8 figs., Strasbourg.

BRITO, I. M. -1965-A - Novos Microfösseis Devonianos do Maranhão. Esc. Geol. Un. Bahia, publ. av. 1, 4 p., 1 est., Salvador.

-1965-B- Nota Prévia sobre os Microfósseis Devonianos de Pernambuco. Esc. Geol. Un. Bahia, publ. av. 3,8 p., 3 est., Salvador.

-1967-A - Silurian and Devonian Acritarcha from Maranhão Basin, Brazil. Micropaleontology, 13, 4, p. 473-482, 2 est., New York.

-1967-B- Novo subgrupo de Acritarcha do Devoniano do Maranhão. An. Acad. Brasil. Cienc., V. 39, n. 1, p. 163-166, 3 est., Rio de Janeiro.

-1976 - Contribuiçāo ao Conhecimento dos Microfósseis Devonianos de Pernambuco. II - Alguns Acritarcha Comuns aos do Devoniano do Saara. An. Acad. Brasil. Cienc., V. 48, n. 4, p. 747-756, 7 figs., Rio de Janeiro.

DAEMON, R. F. - 1964 - Palinomorfos Guias do Devoniano Superior e Carbontfero Inferior das Bacias do Amazonas e do Parnatba. An. Acad. Brasil. Cienc., V. 46, n. 3/4, p. 547-587, 8 est., 4 figs., Rio de Janeiro.

DAEMON, R. F., QUADROS, L. P. \& SILVA, L. C. -1967-Devonian Palynology and Biostratigraphy of the Parand Basin. Bol. Paranaense Geoc., 21-22, p. 99-132, 4 est., Curitiba.

JARDINÉ, S. -1972 - Microplancton (Acritarches) et limites stratigraphiques du Silurien Terminal au Dévonien Supérieur. 7 éme Congr. Int. Strat. Géol. Carbonif., 1, p. 295-311, 3 est., Krefeld.

LUDWIG, G, \& MÜLLER, H. - 1968 - Zur Frage der präkarbonishen Diskordanz im Maranhão-Tucano und Jatoba Becken (Brazilien). Geol. Jb., 85, p. 497-516, 1 est., 7 fugs., 2 tab., Hannover.

MOREAU-BENOIT, A. -1966-Etude des Spores du Dévonien Inferieur d'Avrillé (Le Fléchay), Anjou. Rev. Micropaleontologie, 8, 4, p. 215-232, 3 est., 3 figs., Paris.

SOMMER, F. W.-1956 - Novas Espécies de Tasmanites do Devoniano do Pará. An. Acad. Brasil. Ciênc., V. 35 , n. 1, p. 51-65, 3 est., Rio dẹ Janeiro.

SOMMER, F. W. \& VAN BOEKEL, N. M. -1966-Revisão das Tasmanaceae Brasileiras. An. Acad. Brasil. Ciênc., V. 38 , n. 1, p. 53-64, 1 est., Rio de Janeiro.

\section{THE BRAZILIAN EOGONDWANIC FLORAL SUCCESSION}

\section{OSCAR RÖSLER}

Departamento de Paleontologia e Estratigrafia

This paper has the objective to present an actualized scheme of the distribution of fossil plant assemblages in the outcroping sediments of the Paraná Basin (Brazil). Based on this scheme some general aspects, including the problem of the Carboniferous-Permian boundary, are briefly discussed.

A review of such distribution was pre- 